\title{
La traduction dans la didactique ou la didactique de la traduction - quelques réflexions sur les programmes universitaires en Pologne
}

\author{
Halina Chmiel-Bożek \\ Faculté de Philologie, Université Pédagogique de Cracovie ${ }^{1^{*}}$
}

Le présent article vise à présenter la didactique de la traduction et avant tout le rôle de la traduction didactique dans les plans d'études de la philologie romane en Pologne. Les démarches des autorités ministérielles polonaises, qui vont de pair avec les solutions européennes présentées, entre autres, dans le CECR, ont pour but de préparer les futurs diplômés aux besoins du marché de travail. L'analyse des plans d'études de quatorze philologies romanes en Pologne, y compris le type de cours, le nombre d'heures et de points ECTS, permet de constater que les solutions universitaires ne sont pas homogènes dans cette matière. La traduction didactique est enseignée seulement par quatre universités au premier cycle d'études et par cinq au deuxième cycle. Malgré les recherches actuelles sur le rôle de la traduction didactique et de la médiation/traduction dans l'enseignement du FLE, cette approche est trop souvent regardée en Pologne comme un exercice archaïque et régressif. Mots-clé : compétences en traduction, traduction didactique, didactique de la traduction, programmes universitaires, programmes d'études, médiation.

En Pologne, à partir des années 90, pour diverses raisons, principalement politiques et économiques, le marché de la traduction se développe considérablement. Vus les besoins croissants du marché, on introduit successivement dans les universités polonaises des cours de traduction, généralement obligatoires, pour les étudiants en études philologiques. Aujourd'hui personne ne s'interroge sur la légitimité de ces cours car personne ne s'interroge sur la question si l'on peut et s'il faut enseigner et apprendre les compétences en traduction. Les recherches en didactique ont déjà confirmé la possibilité ou même la nécessité de ces démarches. Comme l'écrit Piotrowska, la traduction comporte des éléments de compétences et, à ce titre, elle peut être apprise et enseignée avec un effet positif (Piotrowska 2016 : VIII). Les cours consacrés à la traduction constituent une

*halina.chmiel-bozek@up.krakow.pl 
réponse à des besoins pertinents et concrets du marché et, avant tout, aux besoins des étudiants, futurs diplômés, qui seront amenés, plus ou moins régulièrement, à effectuer des traductions dans leur vie professionnelle. Dans le présent article nous allons réfléchir sur la place de la traduction dans la didactique au niveau supérieur en Pologne. Plus précisément, étant donné les dimensions limitées du présent article, en nous penchant sur l'analyse des plans d'études proposés aux étudiants de philologie romane, nous allons analyser avant tout la portée de la traduction didactique dans les programmes universitaires.

\section{Objectifs théoriques}

Notons tout d'abord que les projets du ministère de la Recherche et de l'Enseignement supérieur polonais sont, à ce propos, assez ambitieux comme en témoigne déjà la Résolution $n^{\circ} 494 / 2013$ du Conseil principal de la Recherche et de l'Enseignement supérieur du 13 juin 2013 concernant les résultats d'apprentissage prévus pour certains programmes d'études ${ }^{1}$. Dans l'annexe $\mathrm{n}^{\circ} 3$ du document ministériel visé, nous lisons qu'après avoir terminé le premier cycle universitaire d'études philologiques, le diplômé sait, entre autres, traduire de la langue d'un espace culturel donné vers le polonais et inversement. Quant aux diplômés du deuxième cycle universitaire, conformément au document mentionné, ils savent déjà traduire des textes de niveau avancé vers le polonais ainsi que vers la langue étrangère. Qui plus est, le diplômé est capable de traduire de manière autonome des textes pragmatiques, spécialisés et littéraires de la langue source vers la langue ciblée, à la fois de façon orale et écrite, et en utilisant des outils de traduction spécialisés. En outre, il possède des compétences avancées dans le domaine de la traduction orale et écrite. Enfin, le diplômé connaît et sait appliquer les normes éthiques et professionnelles du comportement du traducteur à l'égard du texte, de l'auteur, du client et du destinataire. Après avoir analysé la liste des compétences en traduction, qui doivent être acquises par les étudiants au cours des études, nous pouvons nous interroger sur la pertinence des projets ministériels, peut-être trop ambitieux, et nous demander s'ils sont réalistes ? Quelle que soit notre réponse à cette interrogation, l'annexe de la Résolution, citée plus haut, montre le rôle et le poids accordés aux compétences en traduction des futurs diplômés².

Voir: http://www.rgnisw.nauka.gov.pl/uchwaly/uchwala-nr-494-2013-rady-glownej-nauki-iszkolnictwa-wyzszego-z-dnia-13-czerwca-2013-roku-dotyczaca-propozycji-wzorcowych-opisowefektow-ksztalcenia-dla-wybranych-kierunkow-studiow.html

${ }^{2}$ L'analyse détaillée des programmes universitaires permet de constater que pour faire atteindre les compétences attendues, si ambitieuses, les autorités universitaires polonaises proposent des cheminements différents. Les solutions adoptées ne sont pas uniformes. Cette constatation concerne à la fois la traduction didactique et la didactique de la traduction (Cf. Chmiel-Bożek 2019). 
Notons que les démarches des autorités polonaises semblent aller de pair avec les solutions déjà proposées par les institutions européennes au début du $\mathrm{XXI}^{\mathrm{e}}$ siècle. Il convient de souligner que dans le Cadre européen commun de référence pour les langues, publié en 2001, apparaît la notion de médiation qui, bien qu'elle soit une notion assez large, englobe beaucoup d'aspects propres à la traduction. Rappelons que, selon les auteurs du document, visé ci-dessus, la médiation constitue l'un des modes d'activités langagières, à côté de la réception, de la production et de l'interaction, qui construit la compétence à communiquer langagièrement du sujet apprenant et communiquant. L'utilisateur de la langue n'exprime pas sa pensée mais il doit jouer le rôle d'intermédiaire entre des interlocuteurs incapables de se comprendre. Parmi les activités de médiation on cite, entre autres, l'interprétation et la traduction. Ajoutons encore que les stratégies de médiation visent à traiter l'information et à trouver un sens équivalent (CECR 2001 : 68). En publiant le CECR, les autorités européennes voient la nécessité de développer la compétence de médiation dans l'espace éducatif européen.

\section{Mise en pratique}

L'introduction et la réalisation, dans la réalité universitaire, des projets théoriques, mentionnés ci-dessus, paraissent difficiles. Plusieurs facteurs peuvent poser différents problèmes aux enseignants et aux étudiants, surtout ceux qui veulent apprendre une autre langue que la langue anglaise ${ }^{4}$. Rappelons qu'à l'heure actuelle en Pologne dans le cas qui nous intéresse le plus, c'est-à-dire dans le cas de la langue française, la majorité des universités polonaises ${ }^{5}$ proposent aux étudiants du premier cycle un enseignement à partir du niveau A1. Cela concerne treize parmi quatorze universités polonaises où il $\mathrm{y}$ a une chaire ou un institut de philologie romane ${ }^{6}$.

\footnotetext{
${ }^{3}$ Les données présentées dans le présent article s'appuient sur les plans didactiques proposés dans l'année académique 2017/2018 aux étudiants dans quatorze universités polonaises où l'on peut étudier la langue française ou la philologie romane. Pour la clarté de notre étude, toutes les sources électroniques utilisées sont citées seulement à la fin de la bibliographie.

${ }^{4}$ D'ailleurs les enseignants de la langue anglaise rencontrent également des difficultés liées, entre autres, aux méthodes qui favorisent la fluidité d'expression et non la correction (Smentek 2011 : 446).

5 Toutes ces universités proposent le premier et le deuxième cycle, sauf l'Institut de langues modernes de l'Université de Białystok qui ne propose que le premier cycle d'études.

${ }^{6}$ L'Institut des Langues Romanes et de Traduction de l'Université de Silésie constitue une exception, en ne proposant des études qu'aux personnes qui ont passé le baccalauréat du français.
} 


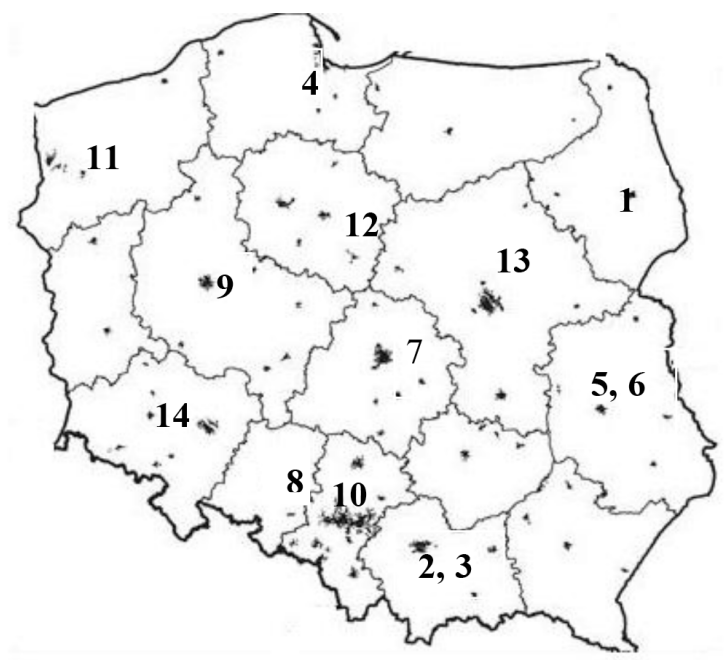

La carte présente des établissements universitaires en Pologne où l'on peut étudier la philologie romane ou la langue française. Dans l'ordre alphabétique des noms de villes, ce sont:

1. Institut de langues modernes de l'Université de Białystok

2. Institut de philologie romane de l'Université Jagellonne de Cracovie

3. Institut de langues vivantes de l'Université de pédagogie de Cracovie

4. Institut de philologie romane de l'Université de Gdańsk

5. Institut de philologie romane de l'Université catholique Jean-Paul II de Lublin

6. Institut de philologie romane de l'Université Marie Curie-Skłodowska de Lublin

7. Institut de philologie romane de l'Université de Łódź

8. Chaire de culture et de langue françaises de l'Université d'Opole

9. Institut de philologie romane de l'Université Adam-Mickiewicz de Poznań

10. Institut des langues romanes et de traduction de l'Université de Silésie

11. Chaire de philologie romane de l'Université de Szczecin

12. Chaire de linguistique et de philologie romane de l'Université Nicolas Copernic de Toruń

13. Institut de philologie romane de l'Université de Varsovie

14. Institut de philologie romane de l'Université de Wrocław

Une grande partie des étudiants commencent l'apprentissage de la langue, à partir du niveau débutant, bien que des groupes soient constitués selon le niveau déjà acquis. Parfois, pour que les études soient attrayantes, il est proposé d'apprendre deux langues. Nous pouvons donc nous poser la question si, dans ces conditions, l'acquisition des compétences avancées en traduction est possible ou si les compétences des futurs diplômés seront suffisantes pour commencer à fonctionner sur le marché des traducteurs/interprètes professionnels ? Rappelons 
que le document ministériel, déjà mentionné, suppose la compétence en traduction non seulement vers la langue maternelle mais aussi vers la langue étrangère. Quelles solutions peut-on donc proposer pour répondre aux besoins du marché concurrentiel de traduction qui nécessite des professionnels capables de réaliser des tâches complexes et hétérogènes?

Pour esquisser les démarches des universités polonaises visant à satisfaire les besoins réels du monde actuel et voir quelle est la présence de la traduction dans les programmes universitaires des quatorze philologies romanes en Pologne, nous avons analysé les programmes du premier et du deuxième cycle qui sont entrés en vigueur pour les étudiants commençant leurs études au mois d'octobre 2017, c'est-à-dire au début de l'année académique 2017/2018. Les données examinées et citées concernent des étudiants qui apprennent seulement une langue, c'est-à-dire la langue française (les cas des sections où l'on enseigne deux langues n'ont pas été analysés).

En premier lieu concentrons-nous sur les propositions adressées aux étudiants du premier cycle. Les cours de traduction sont généralement présents dans les programmes universitaires ${ }^{7}$, quoique leurs dimensions horaires et poids soient variées. Quant aux cours obligatoires, généralement, on peut les diviser en deux parties. La première englobe les matières générales, souvent théoriques et assez restreintes, quant aux heures et le nombre de points ECTS, comme, par exemple, «Introduction à la théorie de la traduction » ou « Bases de l'atelier du traducteur » ou « Introduction à la traductologie ${ }^{8}$.

La deuxième solution, qui nous intéresse le plus, acceptée seulement par une partie des universités polonaises, concerne l'introduction de la traduction, comme cours obligatoire, dans le bloc des travaux pratiques, c'est-à-dire ce que l'on appelle " apprentissage pratique de la langue française ", à côté des autres activités visant à développer les compétences réceptives et productives. Notons à la fois que seulement quatre établissements universitaires sur quatorze le proposent, à savoir l'Institut de philologie romane de l'Université catholique Jean-Paul II de Lublin, l'Université de Łódź, l'Université Adam-Mickiewicz de Poznań et la Chaire de linguistique et de philologie romane de l'Université Nicolas Copernic de Toruń. L'Université catholique de Lublin propose le plus large éventail de possibilités. La totalité de ce cours dure six semestres et équivaut, en somme, à 12 points ECTS. Dans le tableau ci-dessous sont présentées les universités qui dans leurs programmes englobent la traduction dans le bloc des travaux pratiques.

\footnotetext{
L'Institut de la Philologie romane de l'Université de Varsovie constitue une exception, en ne proposant à ses étudiants du premier cycle aucun cours lié à la traduction.

${ }^{8}$ Voir les données sur les sites de l'Institut de langues modernes de l'Université de Białystok, de l’Université Marie Curie-Skłodowska ou l’Université Adam-Mickiewicz de Poznań.
} 


\begin{tabular}{|l|c|c|c|c|}
\hline \multicolumn{1}{|c|}{ Université } & Matière & Semestre & $\begin{array}{c}\text { Nombre } \\
\text { d'heures }\end{array}$ & $\begin{array}{c}\text { Nombre } \\
\text { de points } \\
\text { ECTS }\end{array}$ \\
\hline $\begin{array}{l}\text { Institut de philologie } \\
\text { romane de l'Université } \\
\text { catholique Jean-Paul } \\
\text { II de Lublin }\end{array}$ & $\begin{array}{c}\text { Français } \\
\text { pratique - } \\
\text { traduction } \\
\text { (exercices) }\end{array}$ & $\begin{array}{c}1,2,3,4, \\
5,6\end{array}$ & $30 \times 6=$ \\
180 & $2 \times 6=12$ \\
\hline $\begin{array}{l}\text { Institut de philologie } \\
\text { romane de l'Université } \\
\text { de Łódź }\end{array}$ & $\begin{array}{c}\text { Français } \\
\text { pratique - } \\
\text { traduction }\end{array}$ & 5,6 & $30 \times 2=60$ & $2 \times 2=4$ \\
\hline $\begin{array}{l}\text { Institut de philologie } \\
\text { romane de l'Université } \\
\text { Adam-Mickiewicz de } \\
\text { Poznań }\end{array}$ & $\begin{array}{c}\text { Français } \\
\text { pratique- } \\
\text { traduction }\end{array}$ & 5,6 & $30 \times 2=60$ & $2 \times 2=4$ \\
\hline $\begin{array}{l}\text { Chaire de linguistique } \\
\text { et de philologie } \\
\text { romane de l'Université } \\
\text { Nicolas Copernic de } \\
\text { Toruń }\end{array}$ & $\begin{array}{l}\text { Français } \\
\text { pratique- } \\
\text { traduction }\end{array}$ & 5,6 & $30 \times 2=60$ & $3 \times 2=6$ \\
\hline
\end{tabular}

La traduction dans le bloc des travaux pratiques au premier cycle

La gamme des cours liés à la traduction devient plus riche et diversifiée au premier cycle seulement pour les étudiants qui choisissent, soit certains cours, soit la traduction comme spécialisation. Tel est le cas, avant tout, de la philologie romane à l'Institut des langues vivantes de l'Université de pédagogie de Cracovie, de l'Institut de philologie romane de l'Université de Gdańsk, de l'Université Marie Curie-Skłodowska de Lublin et de l'Institut des langues romanes et de traduction de l'Université de Silésie.

En ce qui concerne le deuxième cycle d'études, la majorité des cours liés à la traduction est offerte aux étudiants qui choisissent, soit la spécialisation, soit le module de traduction. Ainsi la gamme des matières devient-elle variée pour les étudiants de la plupart des établissements universitaires, à savoir de l'Institut de philologie romane de l'Université Jagellonne de Cracovie, de l'Institut de langues vivantes de l'Université de pédagogie de Cracovie, de l'Institut de philologie romane de l'Université Marie Curie-Skłodowska de Lublin et de l'Université de Łódź, de la Chaire de philologie romane de l'Université de Szczecin, de l'Université Nicolas Copernic de Toruń, de l'Institut de philologie romane de l'Université de Varsovie et de l'Université de Wrocław. Notons également que les étudiants qui choisissent l'option pédagogique n'ont généralement guère de cours 
de traduction, ce qui engendre des inconvénients, car comme nous l'avons dit au début, tout le monde, y compris les professeurs de français, sont de temps en temps amenés à traduire ou à se servir de leur compétence de médiation. L'Institut de philologie romane de l'Université de Łódź et la Chaire de culture et de langue françaises de l'Université d'Opole constituent ici une exception car, à côté des cours au choix, tous les étudiants suivent des cours englobant la traduction écrite et l'interprétariat, estimés en somme à 16 points ECTS dans le cas de Łódź et 20 points ECTS dans le cas d'Opole?'

Des cours de traduction dans le cadre de l'apprentissage pratique de la langue, donc obligatoires pour tous les étudiants, sont proposés aux étudiants seulement par cinq universités sur treize qui offrent les études du deuxième cycle. Le tableau ci-dessous présente la liste de ces universités ainsi que la portée de cette formation avec le nombre d'heures et de points ECTS.

\begin{tabular}{|c|c|c|c|c|}
\hline Université & Matière & Semestre & $\begin{array}{l}\text { Nombre } \\
\text { d'heures }\end{array}$ & $\begin{array}{c}\text { Nombre } \\
\text { de points } \\
\text { ECTS }\end{array}$ \\
\hline $\begin{array}{l}\text { Institut de philologie } \\
\text { romane de l'Université } \\
\text { de Gdańsk }\end{array}$ & $\begin{array}{c}\text { Traduction: } \\
\text { langue française }\end{array}$ & 2,4 & $30+15=45$ & $5+3=8$ \\
\hline $\begin{array}{l}\text { Institut de philologie } \\
\text { romane de l’Université } \\
\text { catholique Jean-Paul } \\
\text { II de Lublin }\end{array}$ & $\begin{array}{c}\text { Français pratique } \\
\text { - traduction } \\
\text { spécialisée } \\
\text { (exercices) }\end{array}$ & 3,4 & $\begin{array}{c}30+30= \\
60\end{array}$ & $2+4=6$ \\
\hline $\begin{array}{c}\text { Institut de philologie } \\
\text { romane de l'Université } \\
\text { Adam-Mickiewicz de } \\
\text { Poznań }\end{array}$ & $\begin{array}{c}\text { Français pratique } \\
\text { - traduction }\end{array}$ & $1,2,3,4$ & $\begin{array}{c}30 \times 4= \\
120\end{array}$ & $\begin{array}{c}2+2+4+ \\
4=12\end{array}$ \\
\hline $\begin{array}{c}\text { Chaire de } \\
\text { philologie romane } \\
\text { de l'Université de } \\
\text { Szczecin } \\
\end{array}$ & $\begin{array}{c}\text { Exercices de } \\
\text { traduction: langue } \\
\text { française }\end{array}$ & 1,2 & $15+15=30$ & $2+2=4$ \\
\hline $\begin{array}{c}\text { Institut de philologie } \\
\text { romane de l’Université } \\
\text { de Varsovie }\end{array}$ & $\begin{array}{c}\text { Français pratique } \\
\text { - traduction }\end{array}$ & $1,2,3$ & $30 \times 3=90$ & $\begin{array}{c}4+4+3 \\
=11\end{array}$ \\
\hline
\end{tabular}

La traduction dans le bloc des travaux pratiques au deuxième cycle

9 Notons que l'approche de traduction que l'on adopte à Łódź et à Opole est totalement différente. À Łódź on propose aux étudiants la traduction des textes techniques et professionnels tandis qu'à Opole on se concentre sur la traduction des textes littéraires et culturels. 
Quoique le rôle de la traduction dans les programmes universitaires augmente, on voit que les différences sont considérables. Les universités jouissent dans ce domaine d'une autonomie, d'où toutes ces dissimilitudes, même dans les cas où les exercices de traduction font partie de l'apprentissage pratique de la langue (le décalage, par exemple, entre 4 et 12 points ECTS dans tout le deuxième cycle, comme nous l'avons montré dans le tableau ci-dessus).

\section{3. À la recherche d'une solution}

Malgré les efforts universitaires, la formation des compétences en traduction des acteurs sociaux qui seront censés pratiquer la médiation dans des situations de communication différentes, ainsi que la formation de futurs traducteurs/ interprètes professionnels exigent plus d'effort. Comme l'écrit Płusa, c'est « un processus complexe et coûteux » (Płusa 2007 : 19). Sans doute, ne peut-on pas former un traducteur en 120 heures, mais le nombre de 330 heures dans le cycle semble, lui aussi, insuffisant. C'est pourquoi dans la littérature apparaissent différentes propositions qui visent à combler cette lacune et à résoudre, au moins partiellement, le problème mentionné ci-dessus.

L'une d'elles est liée notamment à la possibilité du renforcement de la traduction dans le processus de l'apprentissage de la langue étrangère. La traduction, autrefois bannie de la didactique des langues étrangères, y compris de la didactique du FLE, à présent y revient en force, bien que les liens entre la didactique et la traduction soient abordés maintenant à partir des présupposés théoriques tout à fait différents, ce qui est accentué dans des publications récentes (Medhat-Lecocq, Negga, Szende 2016 ; Lipińska, Seretny 2016). Dans la littérature consacrée à ce sujet nous rencontrons plusieurs arguments qui soulignent les avantages découlant de l'introduction de la traduction dans le processus d'enseignement et d'apprentissage de la langue étrangère. La traduction y est présentée comme une quintessence de toutes les compétences ou comme la « cinquième compétence langagière » qui développe la communication entre les langues et les cultures (Smentek 2011 : 447). Selon Małgorzata Smentek, par exemple, la traduction est en mesure de répondre à de nombreuses exigences dont la mise en œuvre dépasse les possibilités de l'approche communicative (Smentek 2011 : 447). Cette dernière énumère toute une liste de profits qu'on peut tirer grâce à la traduction (Smentek 2011 : 448). Płusa, à son tour, cite des techniques et des exercices sous-estimés, tels que des exercices de traduction en vue de la consolidation des structures, traduction de mémoire, comparaison de différentes traductions du même texte (Płusa 2007 : 15).

D'ailleurs la traduction didactique et la traduction professionnelle ont beaucoup de points communs dont a déjà parlé, entre autres, Jean Delisle. Les 
dix similitudes énumérées par ce dernier nous permettent de distinguer deux groupes. Le premier concerne généralement les compétences langagières. Or, en traduisant, que ce soit une traduction didactique ou bien professionnelle, on apprend à repérer les difficultés de traduction, à éviter les inférences, on complète son apprentissage par l'acquisition des connaissances sur la civilisation liée à une langue donnée, on apprend à appliquer la méthode de réflexion logique et analytique, en cherchant à reproduire l'articulation d'une pensée, on développe la souplesse langagière. Le second groupe des similitudes se réfère aux stratégies appliquées lors de la traduction, que ce soit la traduction didactique ou la traduction professionnelle. En premier lieu on apprend que la traduction ne s'exerce pas dans une subjectivité pure, mais qu'elle obéit à certaines règles. On apprend donc à pratiquer ces principes, règles et procédés. On utilise aussi le même métalangage (Delisle 1998 : 213).

Vues les similitudes énumérées ci-dessus, on peut constater qu'au moins une partie des objectifs, qu'on veut atteindre, en utilisant la traduction dans la didactique et la didactique de la traduction professionnelle, est la même. Les objectifs de la didactique de la traduction visant à atteindre des compétences professionnelles, quoique sans doute plus vastes, ne se distinguent pas toujours des objectifs des exercices propres à la traduction didactique. Bref, les avantages liés à l'introduction de la traduction dans l'éducation langagière au niveau avancé l'emportent sur tous les contre-arguments. En conséquence, ces deux domaines, au lieu de s'opposer, devraient plutôt se compléter, voire se soutenir mutuellement.

Malheureusement en Pologne les enseignants de langues étrangères, même au niveau supérieur, sont souvent convaincus que l'introduction de la traduction dans la didactique est injustifiée car la traduction constitue une approche archaique qui n'est pas capable de relever les défis posés par le monde moderne de la communication dynamique. Płusa constate que la traduction, considérée à tort comme un composant principal de la méthodologie régressive, a été catégoriquement rejetée (Płusa 2007 : 14). Smentek regrette qu'en Pologne la traduction dans la didactique est fautivement associée à la méthode grammaire-traduction. Durant plusieurs années la traduction dans la didactique est regardée comme un exercice ennuyeux et difficile, long, non-communicatif, ne favorisant que le développement de la grammaire et des compétences en lecture et en écriture (Smentek 2011 : 447). Lipińska et Seretny ajoutent à ce propos que les exercices basés sur la traduction sont souvent perçus comme une manifestation d'ignorance et d'incompétence des enseignants qui les utilisent, ce qui résultent d'un manque de connaissances sur le rôle de la traduction dans la didactique des langues et d'une pénurie de matériel didactique approprié (Lipińska, Seretny 2016 : 9). C'est pourquoi, entre autres, la traduction présente dans le bloc des matières liées à l'apprentissage pratique de la langue, reste toujours en marge. Comme 
nous l'avons vu, seulement quatre établissements universitaires, sur quatorze, l'introduisent au premier cycle d'études et cinq au deuxième cycle études.

Notons enfin que cette méfiance à l'égard de la traduction dans la didactique n'est pas seulement un phénomène polonais. Citons à ce propos l'opinion du chercheur chinois Chen Wei qui explique en quoi consistent les difficultés liées au choix des textes adéquats, au public ciblé, dont les besoins et le niveau de la langue ne sont pas souvent uniformes, ou aux objectifs différents de la traduction didactique et de la didactique de traduction " qu'il est impossible de réaliser en même temps » (Wei 2017 : 46). Qui plus est, ce problème n'a pas été résolu par l'introduction de la notion de médiation dans le Cadre européen commun de référence pour les langues (2001). D’après Ivana Franić, de l'Université de Zagreb, « il semble que certains concepts introduits par le Cadre ne soient pas suffisamment pris en compte dans la pratique. La composante qui nous semble peu exploitée est celle de la médiation » (Franić 2011 : 40). D'ailleurs le fait qu'en 2016, entre autres, le Conseil de l'Europe a publié le volume complémentaire du CECR, entièrement consacrée à la médiation, qui vise à « permettre aux utilisateurs du CECR de mieux comprendre la nature et l'intérêt de la médiation dans l'enseignement (des langues) à tous les niveaux » (North, Piccardo 2016:53) en témoigne le mieux.

\section{Conclusion}

Chen Wei aboutit à la constatation que, malgré toutes les difficultés pour lier « la pédagogie de la traduction » et « la traduction pédagogique» lors du cours de traduction chinois-français, ce cursus est un « mal nécessaire » qui « répond à des besoins pertinents et réellement existants » (Wei $2017: 55$ ). Vu le besoin de communiquer, le rôle des compétences en traduction et en médiation augmente sans cesse. Les efforts des universités, visant à satisfaire ce besoin du marché et du monde globalisé, sont perceptibles en Pologne bien que les programmes d'études concernant la traduction dans la didactique et la didactique de la traduction, loin d'être homogènes, proposent des cheminements différents pour atteindre les résultats attendus. Il n'y a jamais de solutions idéales pour tout le monde et, en élaborant des programmes, les autorités universitaires sont souvent forcées à prendre des décisions définitives pour toute la communauté universitaire. Malgré tous les efforts déjà entrepris, il semble que le développement de la compétence en traduction exige plus de démarches. L'apprentissage et l'enseignement de la traduction ainsi que l'apprentissage et l'enseignement de la langue à l'aide de la traduction ne sont pas faciles surtout dans le cas de la langue française car le manque de matériel didactique approprié est, en Pologne, apparent. Étant donné les dimensions limitées du présent article, nous nous sommes concentrée particulièrement sur l'analyse de la traduction dans la didactique, bien que l'analyse 
détaillée de l'offre des universités concernant la didactique de la traduction puisse mener à des conclusions intéressantes (Chmiel-Bożek 2019). À ce stade de nos réflexions, nous pouvons constater que le temps est venu d'introduire enfin la traduction dans la didactique du FLE. Comme le conclut avec justesse Płusa, toutes les méthodes qui sont efficaces doivent être autorisées lors de l'apprentissage de la langue et de la traduction (Płusa 2007 : 16). Si tout ce qui ne nuit pas au développement est permis et si les didacticiens et même les institutions européennes le soulignent, pourquoi ne pas introduire la médiation/traduction dans le bloc des travaux pratiques du FLE dans toutes les universités polonaises. La traduction didactique mérite sa place dans les programmes universitaires.

\section{Références bibliographiques}

CECR 2001 : Conseil de l'Europe, Cadre européen commun de référence pour les langues : apprendre, enseigner, évaluer, Paris : Didier.

Chmiel-Bożek 2019 : H. Chmiel-Bożek, « Miejsce dydaktyki przekładu w planach nauczania na kierunkach kształcących romanistów w polskich ośrodkach uniwersyteckich », Rocznik Przekładoznawczy, $n^{\circ} 14$, (en cours d'impression).

Delisle 1998 : J. Delisle, H. Lee-Jahnke (dir.), Enseignement de la traduction et traduction dans l'enseignement, Ottawa : Les presses de l'Université d'Ottawa.

Franić 2011 : I. Franić, « Le concept de médiation proposé par le CECR : les représentations des apprentis traducteurs sur la médiation », Synergies Europe, $n^{\circ}$ 6, 39-49. https://gerflint.fr/Base/Europe6/Europe6.html. 03/02/2019.

Lipińska, Seretny 2016 : E. Lipińska, A. Seretny (dir.), Tłumaczenie dydaktyczne w nowoczesnym kształceniu językowym, Kraków: Księgarnia Akademicka.

Medhat-Lecocq, Negga, Szende 2016 : H. Medhat-Lecocq, D. Negga, T. Szende (dir.), Traduction et apprentissage des langues. Entre médiation et remédiation, Paris : Éditions des archives contemporaines.

North, Piccardo 2016 : B. North, E. Piccardo, Cadre européen commun de référence pour les langues : apprendre, enseigner, évaluer. Élaborer des descripteurs pour illustrer les aspects de la médiation pour le CECR, Strasbourg : Conseil de l'Europe.

Piotrowska 2016: M. Piotrowska, Proces decyzyjny thumacza. Zarys metodyki nauczania przektadu, Warszawa: Wydawnictwo C.H.Beck.

Płusa 2007 : P. Płusa, Rozwijanie kompetencji przekładu i kształcenie tłumaczy, Katowice: Wydawnictwo Uniwersytetu Śląskiego.

Smentek 2011 : M. Smentek, « Doskonalenie kompetencji językowej przyszłych nauczycieli języka angielskiego: inne spojrzenie na przekład», in U. Malinowska (dir.) Język - literatura - kultura. Konteksty glottodydaktyczne, Płock: Wydawnictwo PWSZ w Płocku, 445-451.

Wei 2017 : Ch. Wei, « Réflexions sur le cours de traduction chinois-français dans le cadre du programme de français en licence à l'université chinoise », Synergies Chine, $\mathrm{n}^{\circ}$ 12, 43-56. http://gerflint.fr/Base/Chine12/Chine12.html. 03/02/2019. 
Liste de sites universitaires, consultés le 2 février 2019, contenant des plans d'études:

http://neo.uwb.edu.pl/?page_id=13310

http://www.ifr.filg.uj.edu.pl/filologia-romanska-i-stopnia

http://neofilologia.up.krakow.pl/?page_id=299

https://fil.ug.edu.pl/wydzial/instytuty_i_katedry/instytut_filologii_romanskiej/biezacy_ rok_akademicki/plan_studiow

http://e.kul.pl/qlprogram.html?ra=0\&etap=0\&kid=499\&op=2

https://www.umcs.pl/pl/programy-studiow,11651.htm

http://romanistyka.uni.lodz.pl/programy.htm

http://romanistyka.wfil.uni.opole.pl/siatki-studiow/

http://neo.amu.edu.pl/ifrom/index.php?option=com_content\&task=view\&id=30\&Item $\mathrm{id}=28$

https://irk.us.edu.pl/irk/application/program?id=33

http://wf.usz.edu.pl/katedra-filologii-romanskiej/strefa-studenta/\#program-studiow

http://www.home.umk.pl/ kfrwww/programy-i-studiow/

http://irom.wn.uw.edu.pl/?page id=28\&lang=pl

https://ifr.uni.wroc.pl/pl/program-studiow-1

Халина Хмјел-Божек

\section{Превођење у дидактици или дидактика превођења: неколико запажања о факултетским програмима у Пољској}

У чланку се испитује дидактика превођења с посебним освртом на улогу дидактичког превода у наставном плану и програму различитих катедри за романистику у Пољској. Одлуке донете на нивоу министарства, које су у складу с европским решењима представљеним, између осталог, у Заједничком европском оквиру за живе језике, имају за циљ да припреме студенте за тржиште рада. Анализа наставних планова четрнаест катедри за романистику у Пољској, укључујући ту и тип часа, укупан број часова и број ЕСПБ, показује да су универзитети ово питање решавали на различите начине. Тако је дидактичко превођење присутно на свега четири универзитета на основним студијама, и на пет универзитета на мастер студијама. Упркос савременим истраживањима о улози дидактичког превођења и медијацији/превођењу у настави француског као страног језика, ова наставна метода се у Пољској често сматра застарелом, па чак и назадном.

Кључне речи: преводличке компетенције, дидактички превод, дидактика превођења, универзитетски програм, наставни план, медијација 